\title{
Raman Fiber Bundle Probe of Individually Filter-Coated Fibers
}

.

\section{Medhanie Tesfay Gebrekidan', Christian Knipfer ${ }^{2}$ and Andreas Siegfried Braeuer ${ }^{{ }^{*}}$}

\author{
${ }^{1}$ Institute of Thermal-, Environmental- and Resources' Process Engineering (ITUN), Technische Universität \\ Bergakademie Freiberg (TUBAF), Freiberg, Germany
}

${ }^{2}$ Department of Oral and Maxillofacial Surgery, University Medical Center Hamburg-Eppendorf (UKE), Hamburg, Germany

\begin{abstract}
We report how we had a Raman fiber bundle probe with individually filter-coated fibers manufactured by organizing an iterative production process between one fiber coating and one fiber bundling company. Short- and long pass filters had directly been deposited at the ends of the excitation and detection fibers, before the single fibers were configured to a 6 -around-1 fiber bundle. We found that the performance of the fiber bundle probe with individually filter-coated fibers, with respect to signal detection and suppression of elastically scattered light, is similar to a free-beam Raman reference probe. The demonstration of a Raman fiber bundle probe with individually filter-coated fibers paves the way for the development of fiber bundle Raman probes suitable for several excitation and detection channels.
\end{abstract}

\section{Keywords}

Raman fiber probe, Optical fiber bundle, Optical filter coating, Raman spectroscopy

\section{Introduction}

The Raman spectrum of a sample originates from an inelastic light/matter-interaction, which is referred to as Raman scattering. The Raman spectrum contains valuable information about the sample, such as its chemical composition [1-3], its temperature [4-6], its polymorphism [7-9], its heterogeneity and - with respect to medicine or biology - also the histopathological state of human, animalistic or herbal tissue [10-12]. Optical fibers play a pivotal role for making Raman measurements possible deep inside the body, remotely or in hardly accessible or hazardous environments $[13,14]$.
In practice, usually the Stokes-part of the Raman spectrum is analyzed. In order to being able to extract as much information as possible from the Raman-Stokes spectrum of a sample, it is advantageous to analyze its entire spectral range between the smallest and the largest potentially occurring Raman shifts. The smallest Raman shift of $0 \mathrm{~cm}^{-1}$ is due to elastic light scattering and therefore corresponds to the wavelength of the excitation laser. The largest potentially occurring Raman shift of approximately $4155 \mathrm{~cm}^{-1}$ corresponds to the intra-molecular vibration of the hydrogen molecule. The largest Raman shifts of other species

*Corresponding author: Andreas Siegfried Braeuer, Institute of Thermal-, Environmental-and Resources' Process Engineering (ITUN), Technische Universität Bergakademie Freiberg (TUBAF), Freiberg, Germany Accepted: December 18, 2021; Published: December 20, 2021

Copyright: (c) 2021 Gebrekidan MT, et al. This is an open-access article distributed under the terms of the Creative Commons Attribution License, which permits unrestricted use, distribution, and reproduction in any medium, provided the original author and source are credited.

Gebrekidan et al. Int J Opt Photonic Eng 2021, 6:044

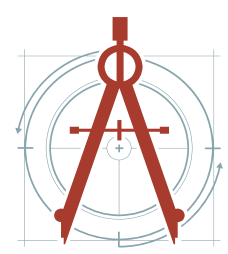


are smaller than $4155 \mathrm{~cm}^{-1}$ and depend on the mass and the bonding of the Raman-active transition of the respective molecules.

Considering an excitation wavelength of $532 \mathrm{~nm}$, the Raman-Stokes spectrum can cover the wavelength range from 532-683 nm, which corresponds to Raman shifts from $0-4155 \mathrm{~cm}^{-1}$. Silicon-based CCD and CMOS detectors feature a high quantum efficiency in this spectral range and thus qualify for the detection of the entire RamanStokes spectrum. If the sample to be analyzed contains species that fluoresce or phosphoresce upon excitation with the Raman excitation laser, their fluorescence or phosphorescence emissions interfere with the desired Raman spectrum. Already spurious concentration of such species cause severe interferences, as the emission processes feature cross sections that, by many orders of magnitude, exceed the small Raman scattering cross sections. The larger the excitation wavelength is, the smaller is the risk of exciting undesired fluorescence or phosphorescence interferences. This explains why many Raman spectrometers use near-infrared (NIR) excitation wavelengths. Considering for example one of the most frequently used excitation wavelengths of $785 \mathrm{~nm}$, the Raman-Stokes spectrum can cover the wavelength range from 785-1165 $\mathrm{nm}$. Unfortunately, siliconbased $\mathrm{CCD}$ and CMOS detectors are rather blind for wavelengths exceeding $900 \mathrm{~nm}$. As a consequence, the reduction of the fluorescence and phosphorescence interferences by using a NIR excitation wavelength is achieved at the expenses of the detectable Raman spectral range. It is true that the information-rich finger print region $\left(0-1800 \mathrm{~cm}^{-}\right.$ $\left.{ }^{1}\right)$ of the Raman spectrum is still detectable with this configuration! But there are many examples, let it be in medicine [15-17] or in process engineering $[3,18,19]$, where most interesting information was extracted not from the finger-print region, but from the spectral range exceeding $1800 \mathrm{~cm}^{-1}$. Remember that this spectral range is not detectable with silicon-based detectors in the case of NIR excitation wavelengths. Intermediate excitation wavelengths in the red spectral range, such as for example one of the emission wavelengths of the HeNe-laser at $632.8 \mathrm{~nm}$, are a compromise. They enable the detection of the Raman spectral range between 1800 and $4115 \mathrm{~cm}^{-1}$, but - due to intensive fluorescence or phosphorescence interferences in the finger-print spectral range - can make the evaluation of the finger-print region challenging [20]. The considerations above illustrate that one would ideally use the $785 \mathrm{~nm}$ excitation wavelength for the detection of a less-interfered finger-print region of the Raman spectrum and temporally decoupled a different excitation wavelength in the red spectral range for the detection of the larger Raman shift region. In order to put this concept into practice in a miniaturized Raman fiber bundle probe, a fiber bundle probe as sketched in Figure 1 a with individually filter-coated fibers would be required. The single-fiber legs labelled "Laser 1" and "Laser 2" would be connected to two different excitation lasers operated at different wavelengths. The fiber bundle legs labelled "Spectrometer 1" and "Spectrometer 2" would be connected to two spectrometers, detecting the Raman spectra excited by the two different excitation lasers. The end of the "Sample leg" would be connected to a lens (system) for focusing the excitation light into the probe volume and for detecting the signal in back-scattering direction into the detection fibers. A potential alignment of the individually filtercoated fibers in the end face of the sample leg is shown. Figure $1 \mathrm{~b}$ shows for comparison a sketch of a state-of-the-art fiber bundle end with central excitation fiber and six surrounding detection fibers. The required filters for the excitation fiber and the detection fibers are coated on a glass rod and a glass tube, which are glued on the fiber bundle end. For details see $[21,22]$ and the patent [23].

The advantages of a fiber bundle Raman probe with individually filter-coated fibers (Figure 1a) are that (i) One Raman probe is compatible with several excitation wavelengths (ii) That the single fibers can be assembled in any configuration in the end face of the sample leg (iii) That one can circumvent the usage of the filter rod and the filter tube (see Figure $1 \mathrm{~b})$ and the accompanying restrictions to the fiber bundle configuration and (iv) That less material interfaces at the fiber bundle ends exist, which can be potential sources of stray-light.

In this manuscript we are not going to demonstrate the usage of various excitation wavelengths with one Raman fiber bundle probe. Instead, we are going to report here how we had a fiber bundle probe with individually filter-coated fibers manufactured and we are going to show its performance relative to a free-beam Raman reference probe. 
(a)

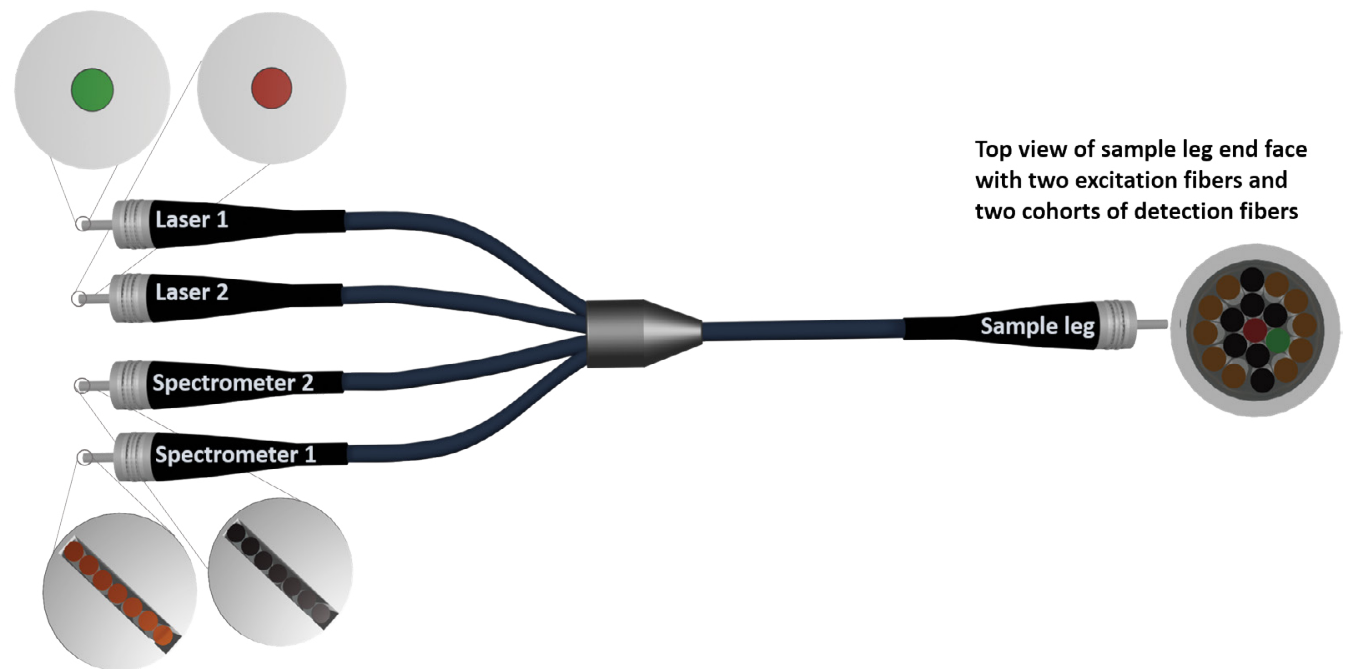

(b)

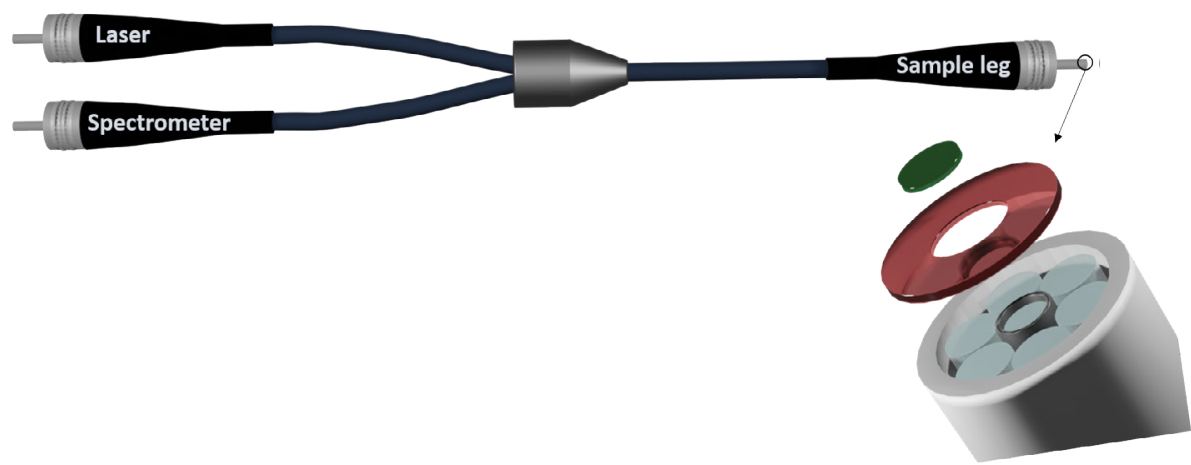

Figure 1: a) Sketch of a concept of a fiber bundle Raman probe with individually filter-coated fibers for two excitation wavelengths; b) Sketch of a state-of-the-art fiber bundle Raman probe for one excitation wavelength (adopted from [22]). The lens (system) for signal generation and detection are not shown.

\section{Raman Probe Development}

Originally, we intended to purchase a fiber bundle with individually filter-coated fibers from one of the internationally operating distributors of optical compounds. Unfortunately, and though being really supportive to us, none of the companies we asked for a quote, was able to even offer such a fiber bundle. It has not been a matter of the price, it has been a matter of the availability. The answer we received was that there is no company or research institution, which in a preconfigured bundle of fibers can coat individual fibers with individual filters. We knew that there are companies capable of coating single fibers or coating fiber bundles with one and the same filter over all the fibers in the bundle. Therefore, we had to identify two companies, one for coating the individual fibers before their assembling to a bundle and one for bundling the already coated individual fibers. The two companies which agreed to support our idea were Omega Optical Inc. (Brattleboro, VT 05301) for filter coating and Art Photonics $\mathrm{GmbH}$ for fiber bundling. Having discussed with both companies the details of our idea, we followed this procedure:

Art Photonics provided the Y-fiber bundle as shown in Figure 2a. Six detection fibers surrounding one excitation fiber proved high detection efficiency [24]. Each fiber featured a length of $4 \mathrm{~m}$, a core diameter of $200 \mu \mathrm{m}$ and a numerical aperture of 0.22 . The spectrometer end was configured with SMA connector and linear fiber alignment to match the geometry of the entrance slit of the spectrometer. The laser end was also preconfigured with SMA connector. The Raman-probe end was not preconfigured and consisted of seven loose single fiber ends.

This Y-fiber bundle was shipped to Omega Optical Inc. for coating of the fibers. For the details of the technology of the deposition of optical filters at the tip of individual fibers it can be referred to the work of Barton, et al. [25]. The six detection fibers were coated with a $>785 \mathrm{~nm}$ long pass filter with an optical density of minimum $3 @ 785$ nm on both 
(a)

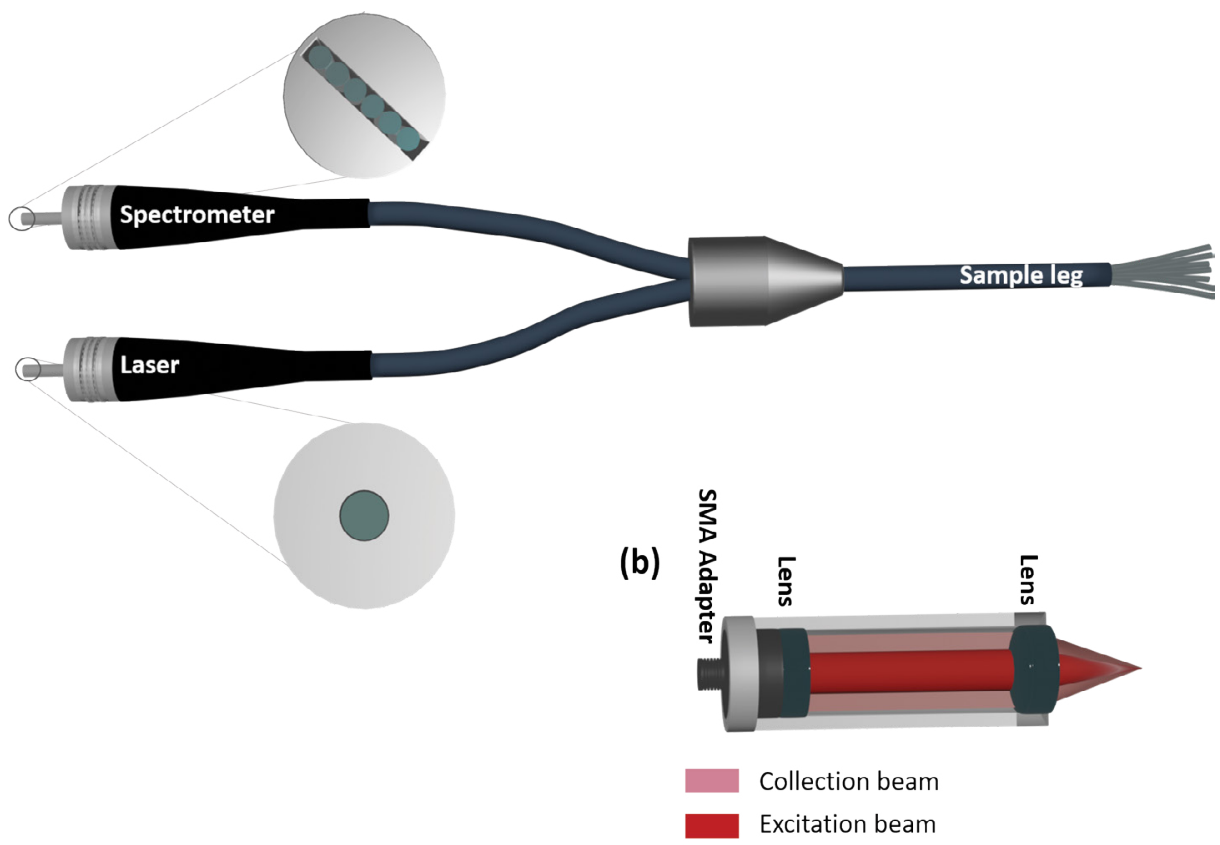

Figure 2: a) Sketch of half-finished Y-fibre bundle, prepared by Art photonics, with spectrometer and laser legs pre-configured with SMA connectors. The Raman-probe end (sample leg) consists of seven loose single fiber ends; b) Sketch of lens (system) consisting of two one inch lenses for focusing the excitation laser light into the probe volume and for collecting the emerging signals in back-scattering direction.

ends; the already preconfigured spectrometer leg end and the loose end of the sample leg. Coating both ends of the detection fibers guarantees an optical density of minimum $6 @ 785$ nm and ensures efficient blocking of the elastically scattered light. The excitation fiber was coated with a $<786 \mathrm{~nm}$ short pass filter only on the loose Raman probe end in order to block the laser-light-induced fiber background at wavelengths exceeding $785 \mathrm{~nm}$ [26].

The coated fiber bundle probe was then shipped back to Art Photonics for the final finishing/ bundling of the Raman probe end in an SMA connector. The excitation fiber was situated in the center, surrounded by the six detection fibers. It is advantageous to skip the usage of glue for assembling the already coated fibers, simply because glue residues cannot be polished from the already coated fiber tips without destroying the filter-coating. Therefore, Art Photonics used aluminum-coated fibers, which can be efficiently assembled by a glue-less sintering process.

\section{Experimental Result}

The Y-fiber bundle probe with individually coated fibers was tested for its performance at Technische Universität Bergakademie Freiberg, Germany, using as excitation source a tunable diode laser
(Sacher Lasertechnik Group) operated at $785 \mathrm{~nm}$ and with $150 \mathrm{~mW}$. For signal detection we used a QE-Pro Ocean-Optics spectrometer equipped with a $100 \mu \mathrm{m}$ slit and operated with a signal integration time of $100 \mathrm{~ms}$. The Raman probe end of the fiber bundle was connected to an assemble of two one-inch lenses aligned in a tube, for focusing the excitation laser into the probe volume and for detecting the scattered light in the vicinity of the laser excitation focus (see Figure 2b). We took a cuvette filled with liquid ethanol as sample and recorded the Raman spectrum of ethanol.

Figure 3 shows a sketch of an also self-engineered free-beam Raman probe. It served as a reference, as the excited volume (excitation beam focus) and the detected volume (volume imaged onto the detection fiber interface) can be aligned congruent, because of what very efficient signal detection is guaranteed. The short-pass filter in the excitation path and the long-pass filter in the detection path are integrated in the free-beam paths. The excitation light and the detected light paths are combined and separated using a dichroic mirror. The excitation fiber features a core diameter of $200 \mu \mathrm{m}$ and a numerical aperture of 0.22 . A round to linear fiber bundle (six-around-one) is used for guiding the detected signal from the free-beam 


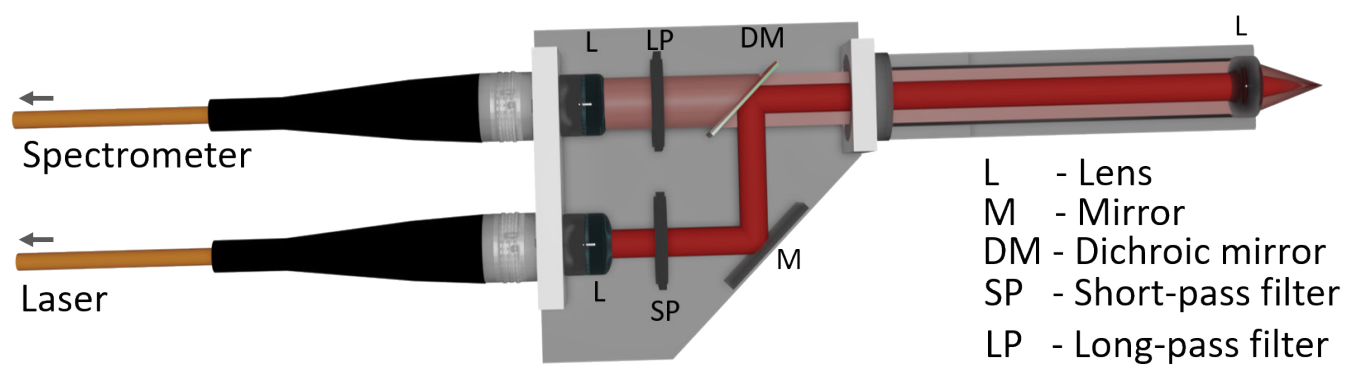

Collection beam

Excitation beam

Figure 3: Sketch of the free-beam Raman probe which served as reference.

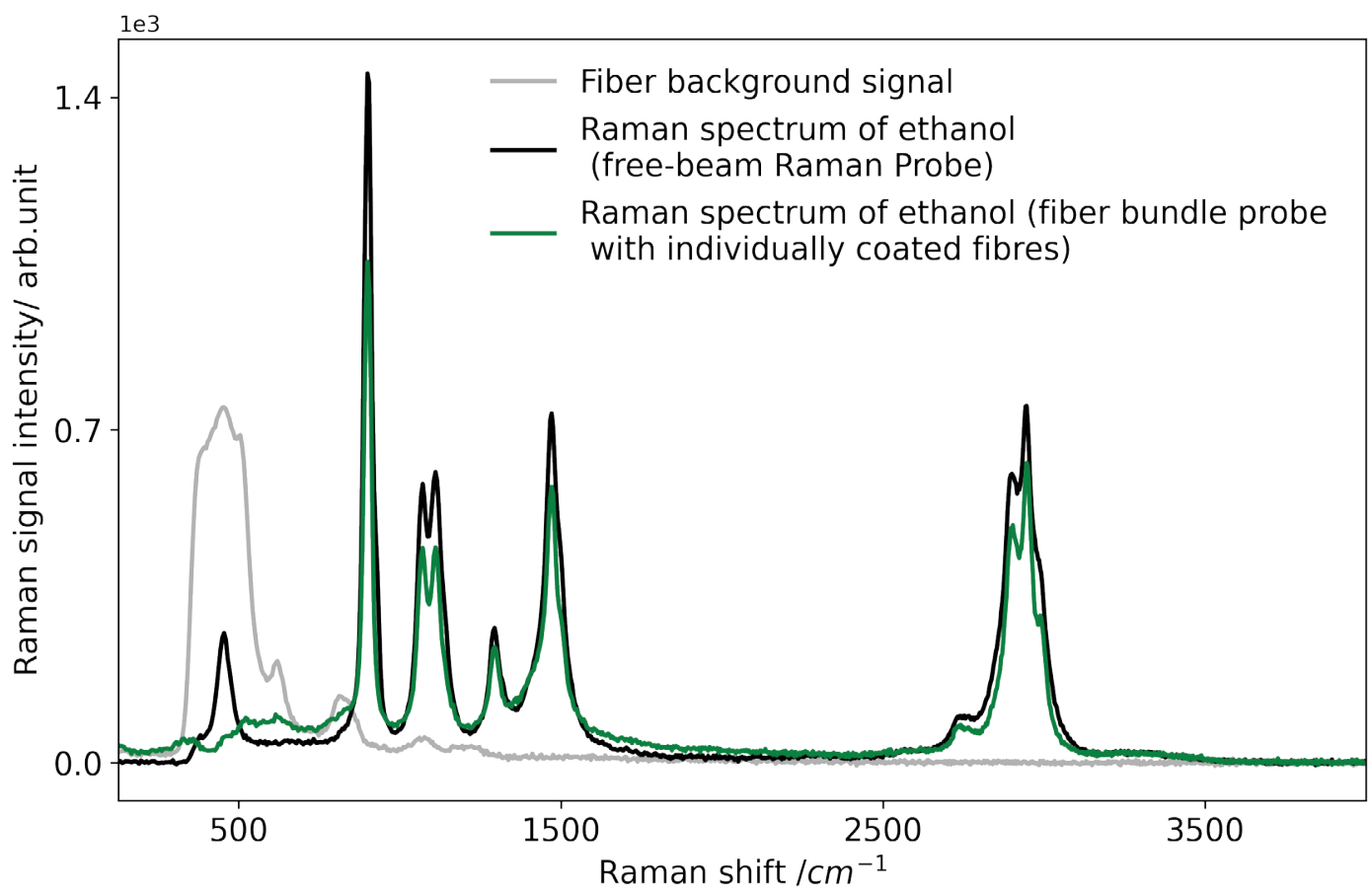

Figure 4: Raw Raman spectrum of liquid ethanol acquired with the Raman fiber bundle with individually filtercoated fibers (green line) and the free-beam reference Raman probe (black line). Fiber background is shown as grey spectrum.

Raman probe (round alignment of detection fibers) to the spectrometer (linear alignment of detection fibers), where each fiber of the bundle features a core diameter of $200 \mu \mathrm{m}$ and a numerical aperture of 0.22 .

The two Raman probes we compare here, the Y-fiber bundle probe with individually coated fibers and the free-beam Raman reference probe, are both operated with the same laser and laser settings, and with the same spectrometer and spectrometer settings. The numerical aperture of both probes is 0.22 , limited by the numerical aperture of the fibers used.

Figure 4 shows raw Raman spectra of liquid ethanol acquired with the fiber bundle probe with individually coated fibers and with the freebeam Raman reference probe. The grey spectrum shows the red-shifted part of the laser-induced background of the $2 \mathrm{~m}$ long excitation fiber of the free-beam Raman reference probe. It can be acquired when detecting the outcome of the demounted excitation fiber with the free-beam Raman reference probe. Then, the excitation beam does not pass the short-pass filter and the long- 
pass filter blocks the excitation wavelength itself and the blue-shifted (with respect to the excitation wavelength) part of the laser-induced background spectrum.

As the pattern of the excitation fiber background is not visible in neither of the spectra acquired with the two Raman probes, we conclude that the short-pass filter efficiently suppresses the fiber background and transmits the excitation wavelength. Obvious differences of both spectra are that the reference probe detects more (but not significantly more) signal, which is expressed by the larger peaks, and also is capable of detecting the ethanol Raman peak at approximately $480 \mathrm{~cm}^{-1}$. The long-pass filter coating of the fiber bundle probe suppresses this peak but transmits the remaining peaks. The shapes of the peaks and also the course of the background are similar. Considering the excitation power of only $150 \mathrm{~mW}$ and the signal integration time of only $100 \mathrm{~ms}$, the performance of both probes is very good, which is expressed in quasi noise-free spectra, with the performance of the fiber probe with individually coated fibers being in the range of the performance of the free-beam Raman reference probe. However, in free-beam Raman probe, the need for external filters at the end of the excitation and detection fibers hinders miniaturization of Raman probe due to added extra bulk to the Raman probe.

\section{Conclusion}

We conclude that the fiber bundle probe with individually coated fibers shows a performance similar to the free-beam Raman reference probe. As described above, the fiber bundle probe with individually coated fibers can be extended to a version suitable for various excitation wavelengths (see Figure 1a). Then the Raman fiber bundle has to contain two excitation fibers, each coated with the corresponding short-pass filter and two types of detection fibers, one type coated with a long-pass filter for one excitation wavelength and the other type coated with a long-pass filter for the other excitation wavelength. Furthermore, fiber bundle probes can be miniaturized much easier than a free-beam configuration, as no dichroic mirror and no tools for aligning the beams along the optical axis are required. This makes the fiber bundle probe with individually filter-coated fibers interesting for applications were small and robust sensors are required, such as for example for endoscopic tissue characterization in medicine or for minimal invasive process diagnostics in chemical engineering.

\section{Funding}

The project leading to this result has received funding from the Wilhelm Sander-Stiftung, Munich, Germany (Grant 2017.111.1 and 2017.111.2).

\section{Acknowledgement}

The authors acknowledge Phillipp Andreas Bernd Braeuer from Friedrich-Alexander-Universitaet Erlangen-Nuernberg for the debates on the production of fiber bundles with individually filtercoated fibers.

\section{Disclosure}

The authors declare no conflicts of interest.

\section{References}

1. Stratmann, Schweiger G (2002) Fluid phase equilibria of ethanol and carbon dioxide mixtures with concentration measurements by Raman spectroscopy. Applied spectroscopy 56: 783-788.

2. Kojima JJ, Hegde UG, Gotti DJ, Hicks MC (2020) Flame structure of supercritical ethanol/water combustion in a co-flow air stream characterized by Raman chemical analysis. The Journal of Supercritical Fluids 166: 104995.

3. Fechter MH, Braeuer AS (2020) Vapor-liquid Equilibria of mixtures containing ethanol, oxygen, and nitrogen at elevated pressure and temperature, measured with in situ Raman spectroscopy in Microcapillaries. J Chem Eng Data 65: 3373-3383.

4. Morawietz T, Marsalek O, Pattenaude SR, Streacker LM, Ben-Amotz D, et al. (2018) The interplay of structure and dynamics in the Raman spectrum of liquid water over the full frequency and temperature range. The Journal of Physical Chemistry Letters 9: 851-857.

5. Bahr LA, Fendt $P$, Pang $Y$, Karl J, Hammer $T$, et al. (2018) Temperature determination of superheated water vapor by rotational-vibrational Raman spectroscopy. Opt Lett 43: 4477-4480.

6. Yang C, Tang H, Magnotti G (2020) High-speed 1D Raman analyzer for temperature and major species measurements in a combustion environment. Opt Lett 45: 2817-2820.

7. Bahr LA, Dousset T, Will S, Braeuer AS (2018) In situ analysis of aerosols by Raman spectroscopy-Crystalline particle polymorphism and gas-phase temperature. Journal of Aerosol Science 126: 143-151. 
8. Braeuer PA, Bahr LA, Röhricht M-L, Schmidt M, Will S (2020) Spatially-resolved crystallinity determination of polymer welding seams by Raman-microscopy. Procedia CIRP 94: 796-801.

9. Liu X, Smith M, Tse S (2010) In situ Raman characterization of nanoparticle aerosols during flame synthesis. Applied Physics B 100: 643-653.

10. Matthies L, Gebrekidan MT, Tegtmeyer JF, Oetter N, Rohde M, et al. (2021) Optical diagnosis of oral cavity lesions by label-free Raman spectroscopy. Biomed Opt Express 12: 836-851.

11. Huang C-C (2016) Applications of Raman spectroscopy in herbal medicine. Applied Spectroscopy Reviews 51: 1-11.

12.Kendall C, Day J, Hutchings J, Smith B, Shepherd N, et al. (2010) Evaluation of Raman probe for oesophageal cancer diagnostics. Analyst 135: 3038-3041.

13.McGregor HC, Short MA, Lam S, Shaipanich T, Beaudoin EL, et al. (2018) Development and in vivo test of a miniature Raman probe for early cancer detection in the peripheral lung. J Biophotonics 11: e201800055.

14.Basu S, Hou H, Biswas D, Daniels-Race T, Lopez M, et al. (2017) Single fiber surface enhanced Raman scattering probe. Journal of Vacuum Science \& Technology B 35: 06GF01.

15.Koljenovic $S$, Schut TCB, Wolthuis $R$, Jong $B$ de, Santos L, et al. (2005) Tissue characterization using high wave number Raman spectroscopy. J Biomed Opt 10: 031116.

16.Barroso E, Smits R, Bakker Schut T, Ten Hove I, Hardillo J, et al. (2015) Discrimination between oral cancer and healthy tissue based on water content determined by Raman spectroscopy. Anal Chem 87: 2419-2426.

17.Desroches J, Jermyn $M$, Pinto $M$, Picot $F$, Tremblay $M-A$, et al. (201) A new method using Raman spectroscopy for in vivo targeted brain cancer tissue biopsy. Scientific Reports 8: 1-10.

18.Bassing D, Braeuer AS (2021) The influence of temperature and pressure on macro-and micromixing in compressed fluid flows; mixing of carbon dioxide and ethanol above their mixture critical pressure. The Journal of Supercritical Fluids 167: 105036.

19. Michelino F, Gebrekidan MT, Zambon A, Vetralla M, Braeuer AS, et al. (2017) In situ Raman-analysis of supercritical carbon dioxide drying applied to acellular esophageal matrix. The Journal of Supercritical Fluids 128: 194-199.

20.Tuschel D (2016) Selecting an excitation wavelength for Raman spectroscopy. Spectroscopy 31: 14-23.

21. Motz JT, Hunter M, Galindo LH, Gardecki JA, Kramer $J R$, et al. (2004) Optical fiber probe for biomedical Raman spectroscopy. Appl Opt 43: 542-554.

22.Agenant M, Grimbergen M, Draga R, Marple E, Bosch $R$, et al. (2014) Clinical superficial Raman probe aimed for epithelial tumor detection: phantom model results. Biomed Opt Express 5: 1203-1216.

23. Marple E (2012) Filtered fiber optic probe. US Patent, Patent No: US 8,175.423 B2.

24. Ross CA, MacLachlan DG, Smith BJ, Beck RJ, Shephard JD, et al. (2020) A miniature Fibre-optic Raman probe fabricated by ultrafast laser-assisted etching. Micromachines 11: 185.

25.Barton J, Carver G, Chanda S, Locknar S, Gupta M (2020) Fiber bundles with integrated bandpass and notch filters for in-vivo Raman spectroscopy. Optical Fibers and Sensors for Medical Diagnostics and Treatment Applications XX 112330S.

26. May J, Li Y-S (1996) Fiber Raman background study and its application in setting up optical fiber Raman probes. Appl Opt 35: 2527-2533. 